\title{
Cavitation Measurement in a Centrifugal Pump
}

\author{
Nikolett Fecser, István Lakatos
}

Széchenyi István University, Egyetem tér 1, 9026 Győr, Hungary

fecser.nikolett@sze.hu; lakatos@sze.hu

Abstract: One of the causes of centrifugal pump instability lies in the phenomenon of cavitation. Cavitation in the centrifugal pump can produce undesirable effects such as deterioration in hydraulic performance. In order to prevent the emergence of cavitation, it is required to know the onset point of the cavitation, in the pump. Our study presents the process and result of the cavitation measurement performed on a centrifugal pump in a closed loop test system. In this study, we applied CFD analysis, which serves as a means of measuring flow in the impeller of the centrifugal pump. The results gained from CFD analysis correspond, approximately, to the measured results.

Keywords: centrifugal pump; cavitation; NPSH; CFD

\section{Introduction}

Water is a determinative and vital element of human life. Therefore, its movement from the place of extraction to the consumer is an important task, and seeking solutions for it has been an issue for all of human development. The issue of water supply has reasonably been a complex task spanning thousands of years, which still has relevance in our current times. Water pumps determine nearly all aspects of our life directly or indirectly. Some of the areas in which water pumps play a significant role are, non-exhaustively, water supply, activities related to water management, health care, crisis and disaster management, firefighting, agriculture, industry, wine sector, energy production, building engineering, food industry, etc.

Water transportation systems are the critical elements of an infrastructure, the operation is also important from the aspect of security, therefore, the uninterrupted, trouble free, transportation, is of great importance. This demand is influenced by several factors, such as uninterrupted, steady, or irregular water consumption, as well as regular or irregular change in the flow characteristics of the transported water.

Cavitation occurring in pipelines, water transportation systems, and more typically, in water pumps, shut-off and regulating valves could be damaged due to this phenomenon. On one hand, it exerts noise and vibration effects on the 
environment and, on the other hand, generates physical damage to the machinery. Harmful vibrations during cavitation also damage the other related equipment and affects the overall operation, negatively.

Based on the data available in scientific literature and the foregoing research results, we can draw the conclusion that in the years between 1960 and 2000, this topic was more extensively dealt with, then subsequently less and less timely research explored this area, with a lesser consideration of the current practice [1$6]$.

In the course of our research work, we observed that there were few laboratories with measurements on this subject, as well as, accessible measurement results. With regard to measurement methods, we also encountered a number of difficulties, in the field of measuring device development and the elaboration of related measuring methods for instance.

\section{The Phenomenon of Cavitation}

On the grounds of the Knapp-Daily-Hammitts definition, cavitation occurs when bubbles grow in a static or flowing fluid and a collapse process follows this growth. If the collapse of the bubbles does occur, then due to the outgassing and formation of vapor bubbles characterizing the growth process, the phenomenon of bubbling or boiling occurs. On this basis, we distinguish gaseous and vaporous cavitation, which differ in their damaging effect on the material. The occurrence of gaseous cavitation is prior to the vaporous cavitation. The bubbles at a certain pressure start to grow awhile due to the dissolved gases from the fluid. As they reach a critical size, their static equilibrium resolves, and an explosive growth process starts. This is vaporous cavitation, which has a destructive effect [7].

Due to the damaging effects of cavitation, there has been an increasing demand for elaborating cavitation research. Cavitation can be a source of significant noise and vibration, and even the hydrodynamic characteristics can change. The phenomenon of cavitation can be examined based on these characteristics, as well.

The phenomenon of cavitation can occur for various reasons. We summarized the major underlying causes hereunder:

- High local liquid flow velocity

- Drop in pressure on the suction side

- Increase in geodetic suction head

- Temperature increase in pumped fluid 
The two main types of measuring cavitation are the open and closed-loop test circuits. In open-loop systems the phenomenon of "cavitation detachment", namely when the pressure on the suction side decreases gradually causing a sudden drop in the pump delivery head (characteristic curve breaks down) and damaging effect occurs on the suction side of the machinery, cannot be measured precisely. It is difficult to change the pressure of open-loop equipment on the suction side. In our study, we deal with closed-loop cavitation testing.

\subsection{Cavitation Measurement in Closed-Loop Test Circuit}

Normally, cavitation testing is based on the ISO 9906 Standard and GOST 6134, however, it should be also taken into account which market the pump involved in the measurement is made for. The specifications and requirements of the relevant market are to be considered at all times [8] [9].

The cavitation measurement that we performed consists of the following steps:

- In the course of the measurement, we apply a few volume flow rates. We set at least ten different volume flow rates. These are called operating points.

- We read the values required for the characteristic curves:

- $\quad$ Static / Differential pressure

- Real-time shaft rotation speed

- Electric power / torque input

- Water level head (for cavitation measurements)

- Water temperature

- Environment temperature

- Apart from the above, other values requested by the customer will be measured. Generally, it is sufficient to do this only once per measurement after a 30-minute operation. These are as follows:

- Bearing temperature

- Bearing and/or baseplate vibration

- Acoustic pressure values

- Leechate

In the hydraulic laboratory, the complete characteristic range can be measured on the pump model built into the test circuit. The power output of the machine model itself cannot be too low, meaning a power of 50-100 kW. Its minimal size and method of measurement are regulated by international standards. These models 
strictly require the accurate scale modelling of the effective transition cross sections, and most importantly, that of the most relevant elements in power transformation (impeller, volute, guide vanes, back-plates, and draft tube). Our measurements were performed as follows: we performed the cavitation measurement on 10 operating points, which based on standards and practical experience: three points below the $97 \%$ pump head rise and seven of them from the start-up to the cavitation detachment. The throttle valve at on suction side was closed while on the discharge side was opened. The only change made, compared to the traditional cavitation measurement, was that a feed pump was built in on the suction side (it was necessary due to low water level). As a result, upstream pressure occurred on the suction side, but it did not affect the rest of the measurement. Figure 1 shows the photo of a closed-loop test circuit.

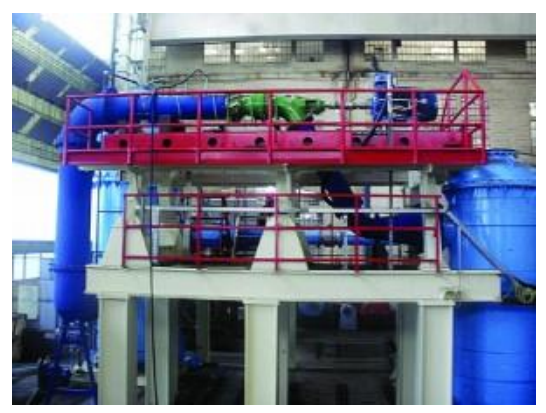

Figure 1

Closed-Loop Test Circuit

Figure 2 illustrates the schematic diagram of a closed-loop test circuit.

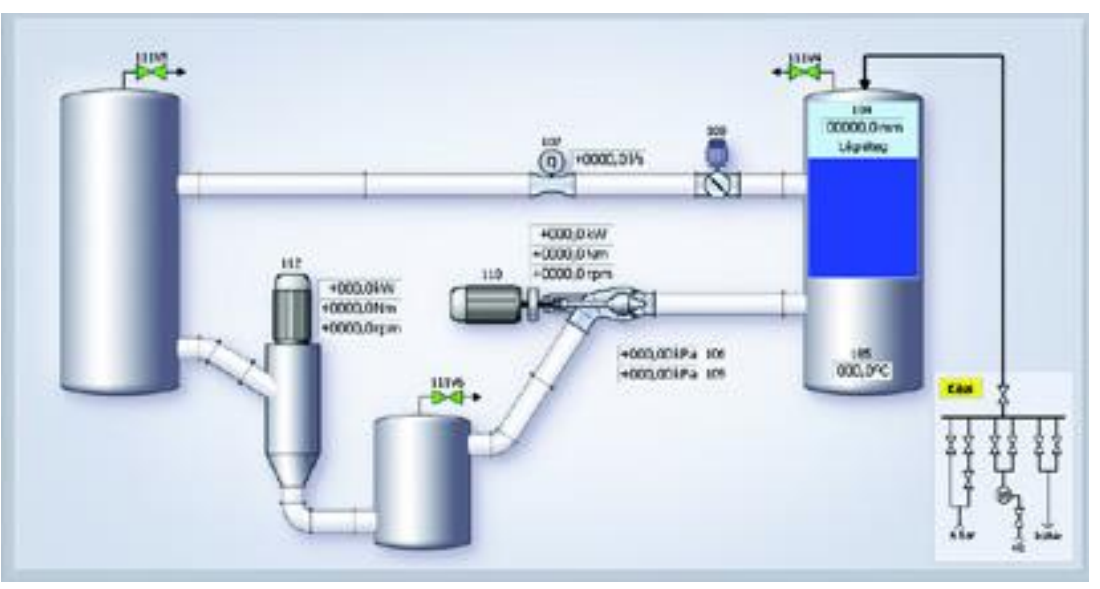

Figure 2

Schematic Diagram of a Closed-Loop Test Circuit 
The measurement results were recalculated from the rated speed, not the real-time values were implemented. It was necessary to have a measurement result that is compatible with the tender curve characteristic submitted to the customer.

In the course of the measurement, we monitored how the results evolved as shown in Figure 3.

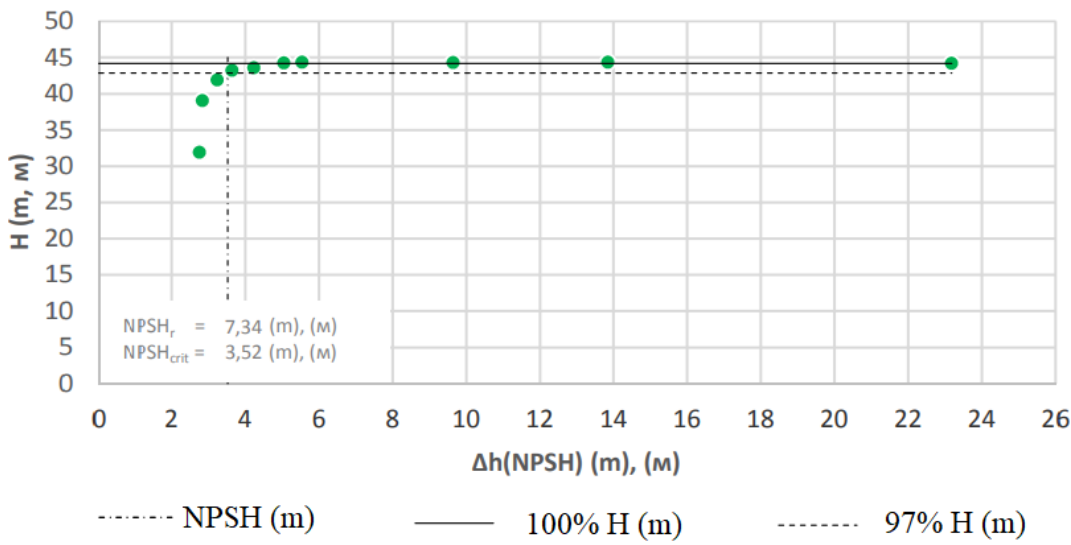

Figure 3

Variation of H-NPSH in the Course of the Measurement

Table 1 demonstrates the calculated data required for the H-NPSH curve of the pump.

Table 1

Calculated Data of the Pump

\begin{tabular}{|l|l|l|}
\hline & \multicolumn{2}{|c|}{ Calculated Values } \\
\hline No. & $\mathrm{H}[\mathrm{m}]$ & $\Delta \mathrm{h}[\mathrm{m}]$ \\
\hline 1 & 44.2 & 23.2 \\
\hline 2 & 44.4 & 13.8 \\
\hline 3 & 44.3 & 9.6 \\
\hline 4 & 44.4 & 5.5 \\
\hline 5 & 44.2 & 5.0 \\
\hline 6 & 43.6 & 4.2 \\
\hline 7 & 43.2 & 3.6 \\
\hline 8 & 41.9 & 3.2 \\
\hline 9 & 39.1 & 2.8 \\
\hline 10 & 32.0 & 2.8 \\
\hline
\end{tabular}

From the recalculated results we determined the $\mathrm{H}-\Delta \mathrm{H}$ (NPSH) curve, which is shown in Figure 4. 


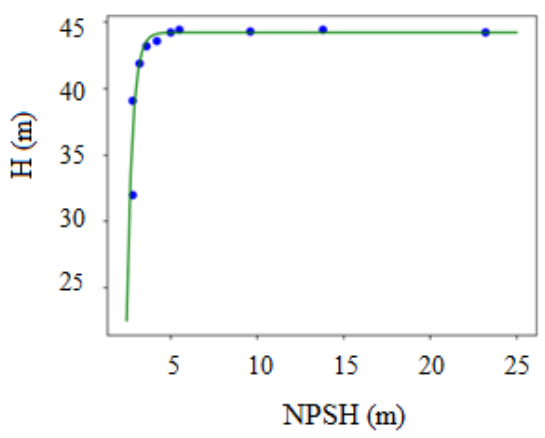

Figure 4

The H- $\Delta \mathrm{H}$ (NPSH) Curve [Authors compilation]

One important characteristic of a centrifugal pump is the NPSH (Net Positive Suction Head). It specifies the minimum pressure at the pump inlet that is required by this particular pump type to operate cavitation-free, meaning the additional pressure, which is required to prevent the fluid evaporation and keep the fluid in the ideal state. From pump aspect the NPSH is affected by the impeller type and the speed of the rotation, whereas from environmental aspect by the fluid temperature, water coverage and atmospheric pressure. The main instruments used in the measurements were:

- Flow rate meter

- Shaft Torque Meter

- Manometer

- Tachometer

An inductive flow meter is installed in the system that ensures accurate and high repeatability flow measurement. Figure 5 shows the measuring scheme of the inductive flow meter.

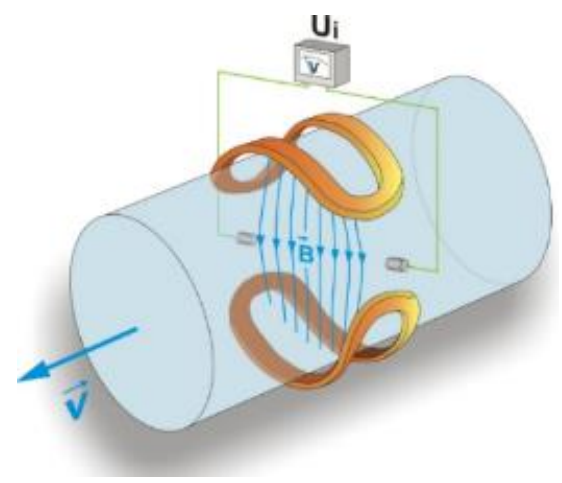

Figure 5

Inductive Flow Meter Measuring Scheme 
The so-called, torque disk is used to measure the shaft power output in a closed loop test circuit.

Figure 6 shows the meter bridge principle, which allows the accurate measurement of slight changes in resistance.

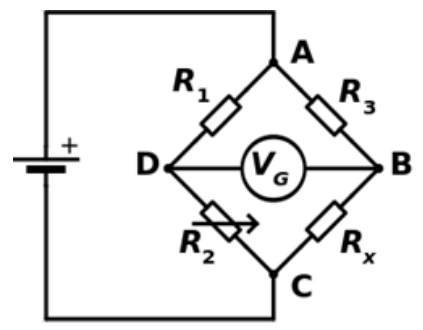

Figure 6

Meter Bridge Principle

In the course of the cavitation measurement, we measured three pressures as the main parameters:

- Suction side pressure

- Differential pressure

- Atmospheric pressure

Figure 7 illustrates the principle of pressure measurement.

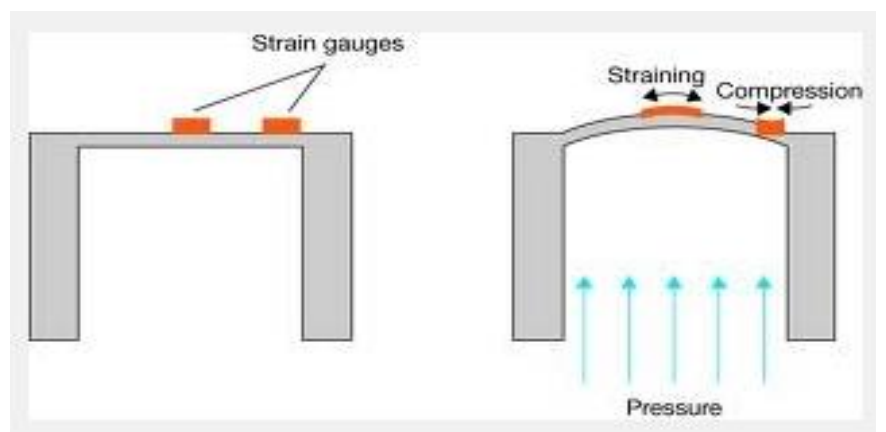

Figure 7

Principle of Pressure Measurement

The speed of rotation is measured by counting the impulses per unit time. The closed loop equipment has an incremental encoder mounted at the end of the motor shaft. It sends 4096 signals per revolution.

Figure 8 shows the measuring principle of the incremental encoder. 


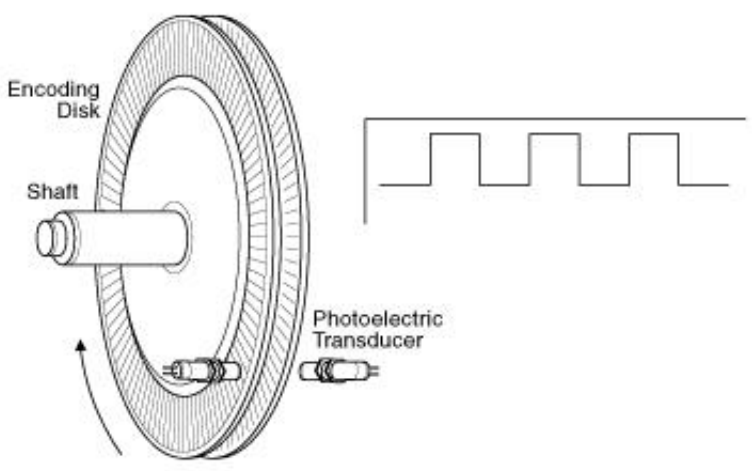

Figure 8

Measuring Principle of the Induction Flow Meter

Figure 9 demonstrates the change of the pressure line in the closed-loop test circuit. By defining one of its points, the line becomes more contoured. At point A, a tank is connected with an air cushion inside. The pressure of the air cushion is kept at a permanent rate by a controller. By gradually reducing the pressure of the air-cushion, the line is sinking in direct ratio. The operating point of the pump remains unchanged until the suction side pressure (specifically NPSH, net positive suction head) is close to the critical value. At this moment, the pump cavitates, its delivery head decreases. In general, we reduce the pressure in 8-10 steps to find the breakdown point. This is cavitation measurement.

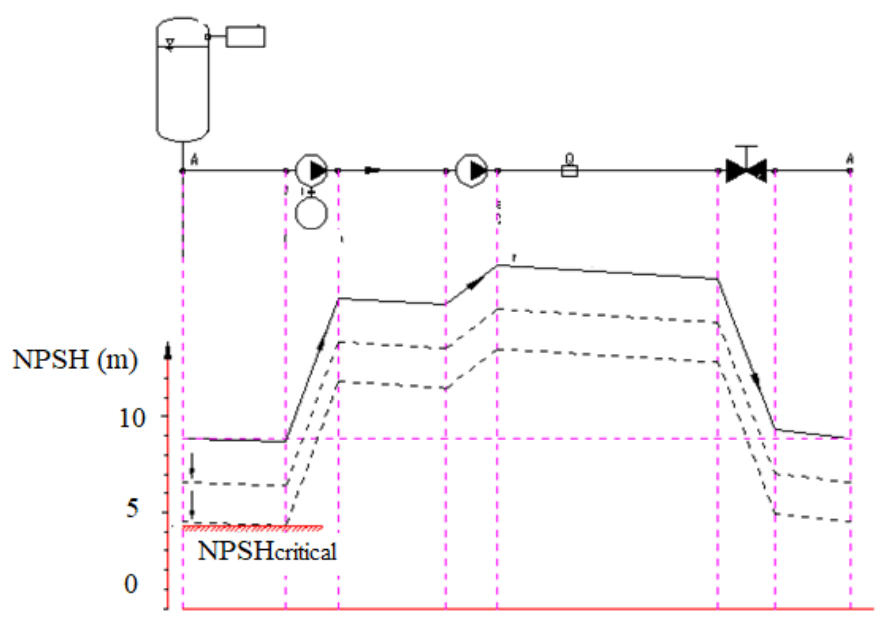

Figure 9

Change of Pressure Line in Closed-Loop Test Circuit 
The pressure valve has a two-stage backpressure regulator. The first (rough) stage performs a huge pressure change in a quick way. The second (fine) stage operates after the first stage ends, and only fine adjustments are needed. Both the first and the second stage regulate between the pressures of 0.2 bars, absolute vacuum, and 2.0 bars, compressed air. The fist (rough) stage operates valves with ON/OFF function. The second (fine) stage adjusts the opening of small sized valves. The vacuum is provided by a pneumatic water-ring pump; the compressed air is supplied by the air network of the factory.

Measurement accuracy is a crucial factor. When performing technical acceptance procedures, one major error in the measurement can hinder the acceptance. When improving, we have to detect even slight changes in efficiency. In a closed-loop test circuit, the dominant source of error is the error of the water volume measurement, which has a rate of $\pm 0.2 \%$. Its decrease therefore is a priority.

Within the resulting measurement error, the measuring accuracy of the efficiency is $\leq \pm 0.35 \%$, and that of the critical cavitation rate is $\leq \pm 1 \%$.

\subsection{Cavitation Model}

\subsubsection{The Description of the Ansys Cavitation Model}

For cavitation modelling, we chose the ANSYS-FLUENT CFD software, which is suitable for multiphase flow modelling [10].

The cavitation model is suitable for determining the cavitation characteristics of water turbines and pumps under vapor formation and condensation with low vapor content due to pressure change [11].

The equation of continuity for vapor phase is as follows:

$\frac{\partial}{\partial t}\left(\alpha \rho_{v}\right)+\nabla \cdot\left(\alpha \rho_{v} v_{v}\right)= \pm \frac{\rho_{v} \rho_{l}}{\rho} \alpha(1-\alpha) \frac{3}{\left(\frac{\alpha 3}{1-\alpha 4 \pi}\right)^{\frac{1}{3}}} \sqrt{\frac{2}{3} \frac{ \pm\left(p_{v}-p\right)}{\rho_{t}}}$

where $\alpha$ denotes vapor volume fraction, $\rho_{\mathrm{v}}$ vapor density, $\mathrm{v}_{\mathrm{v}}$ vapor phase velocity, $\rho_{\mathrm{l}}$ liquid density, $\mathrm{n}$ bubble volume fraction, $\mathrm{p}_{\mathrm{v}}$ saturated vapor pressure (function of temperature), $\mathrm{p}$ mixture pressure, and $\rho$ mixture density.

Three multiphase cavitation models are available in ANSYS-FLUENT software system:

- Singhal model

- Zwart-Gerber-Belamri model

- Schnerr and Sauer model

The first simulation was based on the Singhal model. The results of the simulation and the measured values showed a large difference. We also performed a 
simulation based on the Schnerr and Sauer model, which produced much more accurate results. The Schnerr and Sauer model follows similar approach to the Singhal model in terms of determining the exact expression for the net mass transfer from liquid to vapor.

The Schnerr and Sauer model implements the following standard equations.

The general equation form of vapor volume fraction:

$\frac{\partial}{\partial \mathrm{t}}\left(\alpha \rho_{\mathrm{v}}\right)+\nabla \cdot\left(\alpha \rho_{\mathrm{v}} \overrightarrow{\mathrm{V}}\right)=\frac{\rho_{\mathrm{v}} \rho_{\mathrm{l}}}{\rho} \frac{\mathrm{D} \alpha}{\mathrm{Dt}}$

(2)

where $\alpha$ marks vapor volume fraction, $\mathrm{p}_{\mathrm{v}}$ vapor density, $\rho_{\mathrm{l}}$ liquid density, and $\rho$ mixture density.

$\mathrm{R}=\frac{\rho_{\mathrm{v}} \rho_{\mathrm{l}}}{\rho} \frac{\mathrm{d} \alpha}{\mathrm{dt}}$

$\alpha=\frac{\mathrm{n}_{\mathrm{b}} \frac{4}{3} \pi \mathrm{R}_{\mathrm{B}}^{3}}{1+\mathrm{n}_{\mathrm{b}}^{\frac{4}{3}} \pi \mathrm{R}_{\mathrm{B}}^{3}}$

where $\mathrm{n}_{\mathrm{b}}$ is bubble quantity.

$\mathrm{R}=\frac{\rho_{\mathrm{v}} \rho_{\mathrm{l}}}{\rho} \alpha(1-\alpha) \frac{3}{\mathrm{R}_{\mathrm{B}}} \sqrt{\frac{2}{3} \frac{\left(\mathrm{p}_{\mathrm{v}}-\mathrm{p}\right)}{\rho_{\mathrm{l}}}}$

where $R$ represents the total interphase mass transfer rate per unit volume. $R_{B}$ is the bubble radius.

$\mathrm{R}_{\mathrm{B}}=\left(\frac{\alpha}{(1-\alpha)} \frac{3}{4 \pi} \frac{1}{\mathrm{n}}\right)^{\frac{1}{3}}$

$R_{e}=\frac{\rho_{v} \rho_{l}}{\rho} \alpha(1-\alpha) \frac{3}{R_{B}} \sqrt{\frac{2}{3} \frac{\left(p_{v}-p\right)}{\rho_{l}}}$

where $\mathrm{R}_{\mathrm{e}}$ is a mass transfer source term connected to the growth and collapse of the vapor bubbles.

$$
R_{c}=\frac{\rho_{v} \rho_{l}}{\rho} \alpha(1-\alpha) \frac{3}{R_{B}} \sqrt{\frac{2}{3} \frac{\left(p-p_{v}\right)}{\rho_{l}}}
$$

where $R_{c}$ is a mass transfer source term connected to the growth and collapse of the vapor bubbles.

\subsubsection{Simulation Performed with Ansys Fluent Software}

Using ANSYS-FLUENT v19.1 software system, the simulation of the two-phase cavitation turbulent flow was run on the specified geometry by following the discretization method based on the finite volume method (FVM). The first simulation followed the Singhal model. There was a large difference between the 
simulation and the measured values, for instance, compared to the measured values, a twofold dynamic pressure occurred on the vane leading edge.

Figure 10 shows the spectra of the pressure values on the impeller.

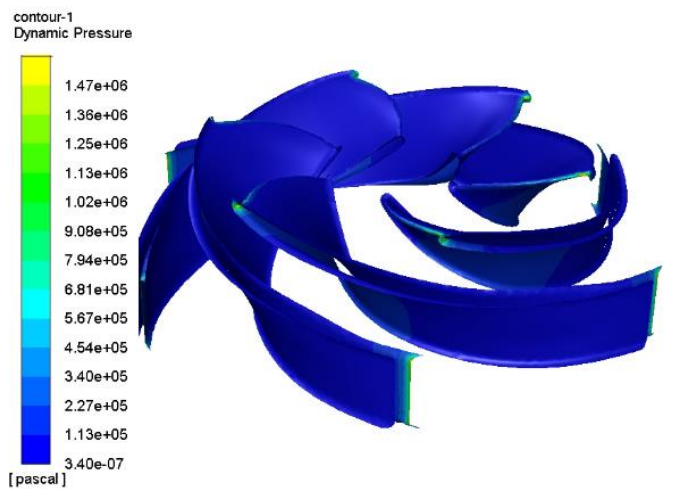

Figure 10

Spectra of Pressure Values on the Impeller [Author compilation]

Figure 11 depicts the velocity rate spectra on the impeller.

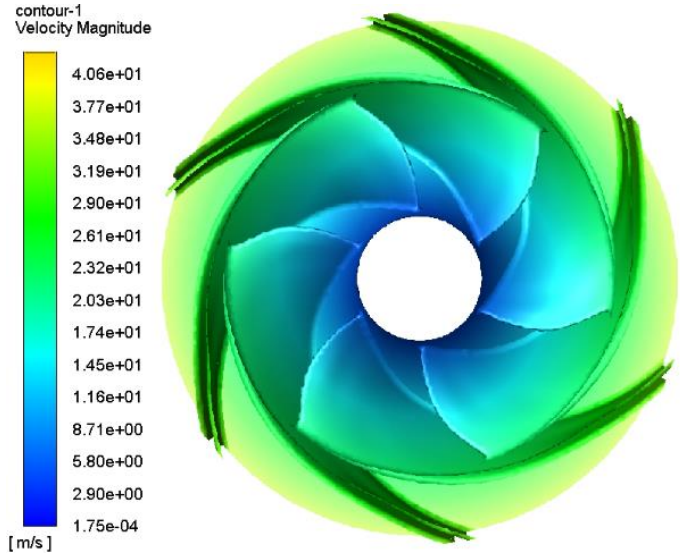

Figure11

Velocity Rate Spectra on the Impeller [Author compilation]

We also performed the simulation based on the Schnerr and Sauer model, which produced much more accurate results. We ran the two-phase turbulent flow with Realizable k-epsilon turbulence model considering standard wall function. In the two-phase flow the primary phase was the liquid and the secondary was the vapor phase. The friction coefficient was calculated by applying the Schiller-Naumann Correlation, whereas the cavitation flow was simulated with the Schnerr-Sauer model. We made the simulation on the assumption of a non-stationary flow. We evaluated the results by using CFD-Post software system. 
Figure 12 presents the spectra of pressure rates on the impeller.

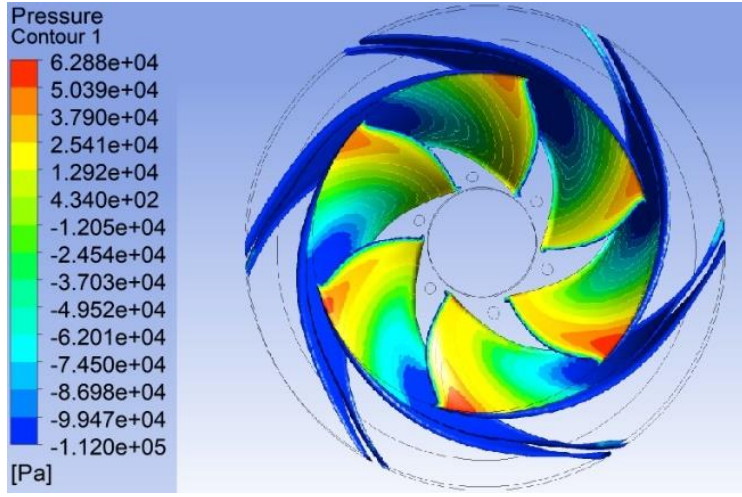

Figure 12

Pressure Rate Spectra on the Impeller [Author compilation]

Figure 13 shows the liquid velocity rate spectra on the impeller.

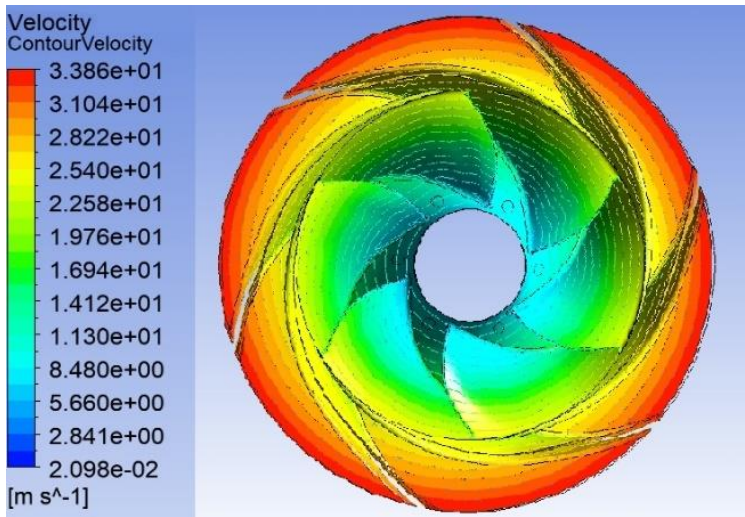

Figure 13

Spectra of Liquid Velocity Rate on the Impeller [Author compilation]

Figure 12 shows the pressure rate spectra on the impeller. The pressure rate is $62.01 \mathrm{kPa}$ on the vane leading edge, $112 \mathrm{kPA}$ on the vane trailing edge. The measured pressure rate on the suction side $-62.5 \mathrm{kPa}$ and on the discharge side $262.3 \mathrm{kPa}$, which indicates that the simulation closely corresponds to the measured values.

Figure 13 depicts the spectra of liquid velocity on the impeller. The liquid velocity on the vane leading edge is $2.52 \mathrm{~m} / \mathrm{s}$, whereas on the vane trailing edge $5.66 \mathrm{~m} / \mathrm{s}$. The values measured on suction and discharge side are around $5.12 \mathrm{~m} / \mathrm{s}$, which also demonstrates that the simulation closely approximates the measured values. 


\section{Conclusions}

Pumps are designed and manufactured so that they operate remotely from the state of cavitation therefore, the factors affecting the initial stage of cavitation are important. Our study dealt with the phenomenon of cavitation occurring in centrifugal pumps. We presented the process and result of a closed-loop cavitation measurement performed with a centrifugal pump in a hydraulic laboratory.

In this study, we used CFD analysis. The results of the CFD analysis are approximately the same as the measurement results. Based on the measurements, we also performed a cavitation simulation that allows to determine the processes occurring during cavitation and the effects on the pump.

Our future goal is to further analyze and refine the simulation results and try to optimize the results obtained. In the course of our research, we contacted several companies and received the information that they applied simulation only in the event of a failure. As a result, we concluded that it would be practical to implement simulations, when measuring at the end of the production, since the results could be beneficial for the subsequent developments and also, filter out the dangerous structural elements of the pump.

We would also like to examine whether there is this relationship between our research and areas of transport science involving on pipeline systems, as a subsystem of transport. In addition to pumps, turbines also play an important role in everyday life. The types of turbines are gas turbines, steam turbines, water turbines and wind turbines, all having different flow mediums. Another important subject of research could be the examination of the cavitation in wind turbines and the related dynamic effects of the air [12-18].

\section{References}

[1] A. A. B. Al-Arabi, S. M. A. Selim, R. Saidur, S. N. Kazi, G. G. Duffy: Detection of Cavitation in Centrifugal Pumps , Australian Journal of Basic and Applied Sciences, 5(10): 1260-1267, 2011 ISSN 1991-8178

[2] M. ČDINA: Detection of cavitation phenomenon in a centrifugal pump using audible sound, Faculty of Mechanical Engineering, University of Ljubljana, Aškerčeva 6, 1000, Ljubljana, Slovenia, Received 26 February 2002, Accepted 25 July 2002, Available online 13 August 2003. DOI:10.1006/MSSP.2002.1514, Corpus ID: 122177370

[3] Tan Lei1, Zhu Bao Shan1, Cao Shu Liang1, Wang Yu Chuan1, Wang Bin Bin: Numerical simulation of unsteady cavitation flow in a centrifugal pump at off-design conditions, First Published December 2, 2013, https://doi.org/10.1177/0954406213514573

[4] S. R. Shah, S. V.Jain, R. N. Patel, V. J. Lakhera: CFD for Centrifugal Pumps:A Review of the State-of-the-Art, Available online 25 April 2013, https://doi.org/10.1016/j.proeng.2013.01.102 
[5] T. Capurso, L. Bergamini, M. Torresi: Design and CFD performance analysis of a novel impeller for double suction centrifugal pumps, Received 9 May 2018, Revised 22 October 2018, Accepted 2 November 2018, Available online $\quad 9 \quad$ November 2018 , https://doi.org/10.1016/j.nucengdes.2018.11.002

[6] Pranav Vyavahare, Lokavarapu Bhaskara Rao, Nilesh Patil: CFD Analysis of Double Suction Centrifugal Pump with Double Volute, Received 26 August 2017; accepted after revision 05 December 2017, https://doi.org/10.3311/PPme.11425

[7] Robert T. Knapp, James W. Daily, Frederick G. Hammitt: Cavitation, McGraw-Hill Book Company, New York, 1970

[8] Rotodynamic pumps. Hydraulic performance acceptance tests. Grades 1, 2 and 3 (ISO 9906:2012), MSZ EN ISO 9906:2013

[9] Rotodynamic pumps. Methods of testing, GOST 6134-87, 2007

[10] ANSYS-FLUENT v19.1 Tutorial guide, 2009

[11] József Nyers, G. Laszló: Analysis of heat pump's condenser performance by means of mathematical model, Acta Polytechnica Hungarica, 2014, ISNN 1785-8860

[12] László Pokorádi: Fluid transport system linear parameter sensitivity analysis, Szolnok Scientific Publications XVII, pp. 43-55, 13 p. (2013)

[13] László Pokorádi, Boglárka Molnár, Monte-Carlo Simulation Analysis of parametric uncertainity of hidraulic system, In: Pokorádi, László (ed.) Technical Science in the Northeast Hungarian Region 2013: conference presentations, Debrecen, Hungary: Debrecen Academic Committee Technical Committee (2013) 518 p. pp. 171-180, 10 p.

[14] László Pokorádi, The uncertainty analysis of the pipeline system, Polytechnical University of Bucharest. Scientific Bulletin. Series D: Mechanical Engineering 73: 3 pp. 201-214, 14 p. (2011)

[15] Moustafa El-Gindy, Hossam Ragheb, Rear wing spoiler effects on vehicle stability and aerodynamic performance, International Journal of Vehicle Systems Modelling and Testing, 2020

[16] Szauter, Ferenc; Péter, Tamás; Bokor, József Complex Analysis of the Dynamic Effects of Car Population Along the Trajectories, In: ASME (szerk.) ASME 2015 International Design Engineering Technical Conferences and Computers and Information in Engineering Conference: Mechatronics for Electrical Vehicular Systems. New York (NY), American Society of Mechanical Engineers (ASME) (2016) Paper: DETC201547075, $6 \mathrm{p}$. 
[17] Szauter, Ferenc; Péter, Tamás; Bokor, József Examination of complex traffic dynamic systems, ACTA TECHNICA JAURINENSIS 6: 2 pp. 1117,7 p. (2013)

[18] Szauter, Ferenc; Peter, Thomas; Bokor, József Investigation of a Complex Transport Dynamics System, In: Péter, Tamás (ed.) Innovation and Sustainable Surface Transport Conference: IFFK 2012 Budapest, BMF, Hungarian Academy of Engineering, (2012) pp. 108-111, 4 p. 